University of Nebraska - Lincoln

DigitalCommons@University of Nebraska - Lincoln

\title{
Configuration of Traffic-Responsive Plan Selection System Parameters and Thresholds: Robust Bayesian Approach
}

Montasir M. Abbas

Texas A \& M University - College Station

Anuj Sharma

University of Nebraska - Lincoln, anujs@iastate.edu

Follow this and additional works at: https://digitalcommons.unl.edu/civilengfacpub

Part of the Civil Engineering Commons

Abbas, Montasir M. and Sharma, Anuj, "Configuration of Traffic-Responsive Plan Selection System Parameters and Thresholds: Robust Bayesian Approach" (2004). Civil Engineering Faculty Publications. 16.

https://digitalcommons.unl.edu/civilengfacpub/16

This Article is brought to you for free and open access by the Civil Engineering at DigitalCommons@University of Nebraska - Lincoln. It has been accepted for inclusion in Civil Engineering Faculty Publications by an authorized administrator of DigitalCommons@University of Nebraska - Lincoln. 
Published in Transportation Research Record: Journal of the Transportation Research Board, no. 1867 (2004): $233-242$.

Copyright 2004, National Academies/National Research Council. Used by permission.

\title{
Configuration of Traffic-Responsive Plan Selection System Parameters and Thresholds: Robust Bayesian Approach
}

\author{
Montasir M. Abbas ${ }^{1}$ and Anuj Sharma ${ }^{2}$ \\ ${ }^{1}$ Texas Transportation Institute, Texas A\&M University, College Station, TX \\ 2 Department of Civil Engineering, University of Nebraska, Lincoln, NE; asharma3@unl.edu
}

Traffic-responsive plan selection (TRPS) is one of the two major closed-loop system modes of operation. The TRPS mode is more beneficial than its competitor (time-of-day mode) because of its ability to accommodate abnormal traffic conditions such as incidents, special events, and holiday traffic. However, no guidelines are available for optimal setup of TRPS systems. Improper configuration of a TRPS system can result in inefficient system performance or unstable operation in which the closed-loop system operates in a perpetual transitioning state. The TRPS mechanism implemented in current traffic signal controllers is described, and a Bayesian-based methodology for selecting an optimal set of TRPS factors, parameters, and thresholds is proposed. The proposed methodology was tested with data from a closed-loop system in Texas and resulted in 100\% classification accuracy.

Coordinating traffic signals in a closed-loop system can provide significant reductions in travel and delay times. A study published in 1997 found that interconnecting previously uncoordinated signals or pretimed signals with a central master controller and providing newly optimized timing plans could result in a travel time reduction of $10 \%$ to $20 \%$ (1). In addition to significantly reducing travel time, properly timed closed-loop systems will also reduce stops, fuel consumption, and vehicle emissions. Another study evaluating the impact of properly timing a closed-loop system in Texas reported a $13.5 \%$ (20.8-million gallons/year) reduction in fuel consumption, a 29.6\% (22-million hours/year) reduction in delay, and an $11.5 \%$ (729-million/year) reduction in stops (2). The study estimated total savings to the public of approximately $\$ 252$ million in the following year alone. These kinds of benefits, however, require the implementation of timing plans that are most suitable to the existing traffic conditions in the field. This operation in tum will require that timing plans be varied in a timely manner as the traffic conditions change.

TYPICAL CLOSED-LOOP SIGNAL SYSTEM COORDINATION MODES

The coordination of traffic signals can be achieved by interconnecting a master controller to a series of traffic signal controllers forming what is known as a closed-loop traffic signal system. The on-street master supervises the individual intersection controllers and issues commands to implement timing plans stored at the local controllers. The master controller can also report detailed information back to a traffic management center using dial-up telephone or other similar communications channels for monitoring purposes.

The two major modes by which timing plans are selected and activated at the controller at any given time are timeof-day (TOD) and traffic-responsive plan selection (TRPS).

\section{TOD Mode}

The TOD mode is the most common mode of operation of closedloop systems. In the TOD mode, a particular timing plan is implemented according to a predetermined time schedule regardless of the existing traffic conditions. TOD mode can provide stable and good performance when traffic patterns are predictable in terms of when and where they occur in the network (3-6). However, for networks in which traffic patterns are not predictable or in which demands shift with time, the TOD mode can cause the signal system to implement plans that are inappropriate for the actual traffic patterns. Another major drawback of the TOD mode is that engineers need to continually update the timing plans so that the plans match the temporal distribution of traffic patterns.

\section{TRPS Mode}

The TRPS mode provides a mechanism by which the traffic signal system is able to select timing plans in real time in response to changes in traffic demand. In the TRPS mode, system detectors are used to measure occupancy and counts in the closed-loop system network. The occupancy and count information is smoothed, scaled (normalized), and then aggregated by multiplying each value by its corresponding detector weight. The master controller keeps track of the aggregated values and continuously compares them with corresponding thresholds. If the new values exceed their corresponding thresholds, the control system selects a different timing plan from a stored library of timing plans.

The TRPS mode has limited implementation nationwide. The city of Milwaukee, Wisconsin, has implemented a TRPS mode in one of their closed-loop systems 
to manage congestion and reduce traffic accidents (7). Even though the study used only two cycle lengths, 90 seconds and 120 seconds, an increase in approach capacity and vehicle speed over system detectors was reported. The study also reported a significant reduction in adjusted frequency of congestion-related intersection accidents.

Another study of two networks in Lafayette, Indiana, found that the TRPS mode reduced total system delay by $14 \%$ compared with the TOD mode for the midday traffic pattern (8). It was also found that the TRPS system reduced total system delay for morning traffic by $38 \%$. However, because of the lack of guidelines on setting up TRPS systems, a fine-tuning process was performed in the laboratory until the TRPS mode behaved as expected. Consequently, the study reported that TRPS had frequently resulted in unexpected time plans changes, which reduced overall system performance (8).

\section{TRPS CONTROL MECHANISM}

Numerous factors and parameters need to be set up correctly for the TRPS mode to function properly. The TRPS control mechanism is explained and each of the TRPS factors and parameters is elaborated on in relation to system detector data.

\section{System Detectors}

The TRPS mode uses information collected from system detectors (occupancy and counts) to measure the traffic conditions in the closed-loop system network. FHWA provided limited guidelines in locating system detectors (9). The general guidelines require that the system detectors be located relatively far from the traffic signal (10). The Indiana study, for example, used 10 system detectors with setback distances greater than 650 feet from the stop line (8).

A common understanding among traffic controller manufacturers, as reflected in their TRPS mechanism design is that system detectors can be categorized into three groups. Each of these categories would serve a different purpose in the TRPS mechanism:

1. Cycle-level detectors. The information from these detectors is used for determining the appropriate cycle level and therefore they should be located near the critical intersection or intersections.

2. Arterial detectors or directionality detectors. The information from these detectors is used to determine the appropriate offset level and therefore they should be placed in the inbound and outbound directions on the arterial.

3. Nonarterial detectors. The information from these detectors is usually used to determine the appropriate split leveland therefore they should be placed on thesidestreets.

\section{TRPS Factors}

Once the occupancy and count data are collected from system detectors, the information is aggregated by means of certain master controller functions using scaling, smoothing, and weighting factors (11-13).

\section{Scaling Factors}

Scaling factors are used to convert occupancy and count data into a combined value that is independent of the value of the approach capacity. The scaled value ranges from $0 \%$ to $100 \%$, which indicates how close the approach is to its capacity. Some literature provides a recommendation to set the values to the highest observed occupancy value for the system detector over a long period of time (14).

\section{Smoothing Factors}

Smoothing refers to producing a weighted average of the occupancy and count data over time to eliminate the effect of short-term fluctuation of traffic patterns. Each controller manufacturer uses a different approach for smoothing data. However, these approaches are generally based on two mathematical functions. The first approach is called filtering. The filtering method calculates the new value of a variable $x$ (e.g., count) by multiplying the difference between the old smoothed value and the newly collected value of the same variable by a smoothing factor and adding the result to the last smoothed value of the variable:

$\overline{\mathrm{x}}_{\text {new }}=\overline{\mathrm{x}}_{\text {old }}+\mathrm{k}\left(\mathrm{x}_{\text {new }}+\overline{\mathrm{x}}_{\text {old }}\right)$

where

$$
\begin{aligned}
\bar{x}_{\text {new }} & =\text { new smoothed value, } \\
\bar{x}_{\text {old }} & =\text { old smoothed value, } \\
x_{\text {new }} & =\text { new raw value, and } \\
k & =\text { smoothing factor. }
\end{aligned}
$$

Smaller values of $k$ give more weight to past data and result in sluggish system response to changes in the variable $x$. However, larger values of $k$ cause the system to be more responsive to changes in data, but that might also lead the system to be more affected by noise in traffic data. Thus, the smoothing factor must be selected to provide maximum responsiveness while maintaining system stability.

The other smoothing approach is to average the values of the variable $x$ over the previous $n$ time intervals. Clearly, the greater the number of previous time intervals used, the less sensitive the smoothed value is to changes.

\section{Weighting Factors}

Each system detector is assigned a weighting factor by which its data are multiplied during the aggregation process. Despite the implication of the name, a weighting factor does not emphasize the importance of an individual system detector, as will be discussed later. Some manufacturers allow assigning different weighting factors to occupancy and counts as well as a weighting factor at the detector itself. Although selection of the weighting factors is crucial to the operation of the TRPS mode, no guidelines have been offered to help in achieving this task.

\section{TRPS Mechanism and Thresholds}

TRPS uses several computational channel (CC) and pattern selection (PS) parameters to arrive at the final selected timing plan. Figure I shows a general TRPS mech- 


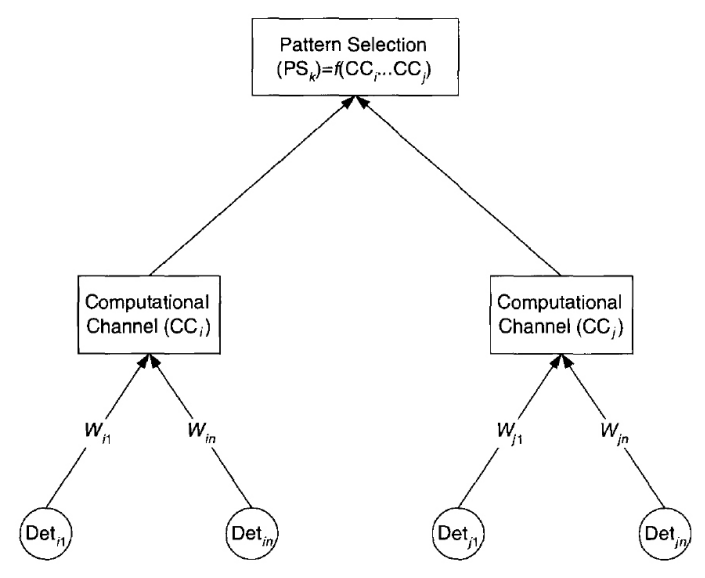

FIGURE 1 General TRPS mechenism.

anism in which occupancy and count information from a group of $n$ system detectors (n differs from one manufacturer to another; e.g., Eagle controllers use eight) is aggregated into a CC parameter (i.e., by multiplying each system detector by its corresponding weight $W$ ). It should be noted that system detectors used with a CC parameter mayor may not be the same system detectors used with another CC parameter. The name and number of CC parameters in a TRPS system differ from one manufacturer to another. Most TRPS manufacturers, however, agree on the names and number of the PS parameters, namely, cycle, split, and offset. Each PS parameter is calculated as a function of several CC parameters. Some of these functions are user selected, whereas others are predefined by the controller manufacturer.

In addition, the TRPS mode requires the operator to predefine entering and exiting thresholds for each PS parameter. The definition of a different entering and exiting threshold provides a hysteresis control, which enhances system stability when the thresholds for each TRPS parameter are set up correctly.

The master controller compares each PS parameter value with its corresponding threshold to identify the appropriate PS level. The three PS levels are used as index values in a table lookup procedure. The lookup-table entries determine which one of the stored timing plans will be selected.

This cycle-split-offset PS parameter nomenclature can be somewhat confusing to the user. Each PS parameter value merely specifies an index to the TRPS lookup table and not the actual cycle, split, and offset values. In addition, it is not necessary to use all PS parameters in the TRPS mechanism. For example, if four timing plans are to be implemented in a closed-loop system and they are differentiable by one PS parameter, then only one PS parameter is needed for TRPS operation. This PS parameter could be anyone of the cycle, split, or offset PS parameters.

As can be deduced from the previous section, setting up a TRPS system to work efficiently is not a trivial task. Besides the possibility of selecting incorrect plans, im- proper values of TRPS parameters can set the system into a perpetual transitioning state. When the system is not in a steady state, benefits of a better timing plan might be offset by the delays associated with the transition between timing plans. Previous research had shown that only marginal benefits could be achieved over TOD operation when fluctuation in traffic demand caused frequent timing plan changes (14). Therefore, there is a need for statistically and theoretically sound guidelines on how TRPS parameters and thresholds can be set up so that TRPS results in optimal and stable system operation.

\section{ROBUST BAYESIAN-BASED APPROACH FOR TRPS SETUP}

The methodology followed in this research was based on the realization that TRPS control is essentially a pattern recognition problem of different traffic conditions, or states. Every intersection approach movement in the closed-loop system is a dimension in the TRPS state space. Variation in the state variable along any of these dimensions can be potentially "sensed" through the occupancy and count information obtained from a system detector placed at that approach. The major challenge of TRPS system setup is the determination of a set of detector weights that can map the multidimensional state space into a unidimensional PS parameter ordinate. This mapping should occur such that maximum separation of different traffic states can be achieved with a set of PS parameter thresholds.

This concept is illustrated in Figure 2, which is a simplified threedimensional space that shows samples from two different state distributions. The reader can think of these two states as low-and high-volume demand cases, respectively. The three-dimensional sample points from these two states correspond to occupancy data from three system detectors placed at three different approaches. Figure $2 \mathrm{a}, \mathrm{b}$, and c correspond to three different sets of detector weights. Figure 2a shows a set of weights that provides poor separation of the two state distributions. Figure $2 \mathrm{~b}$ shows a different set of weights that provides 


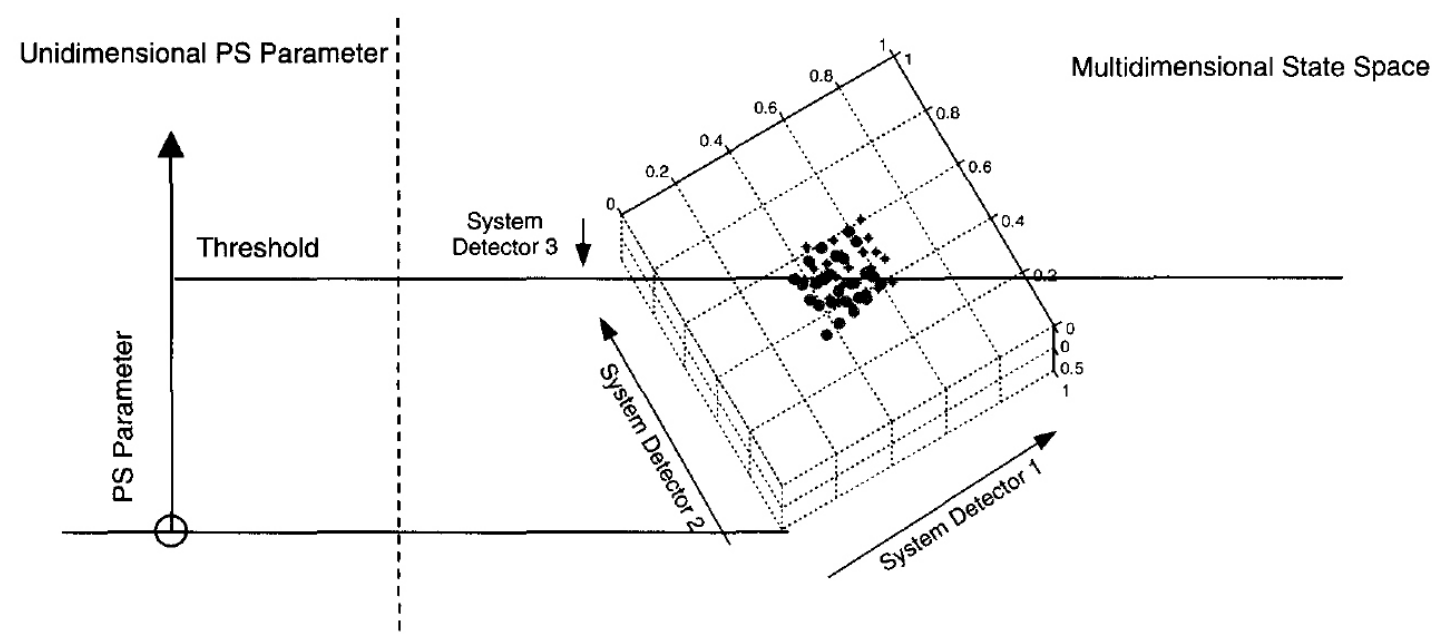

(a)

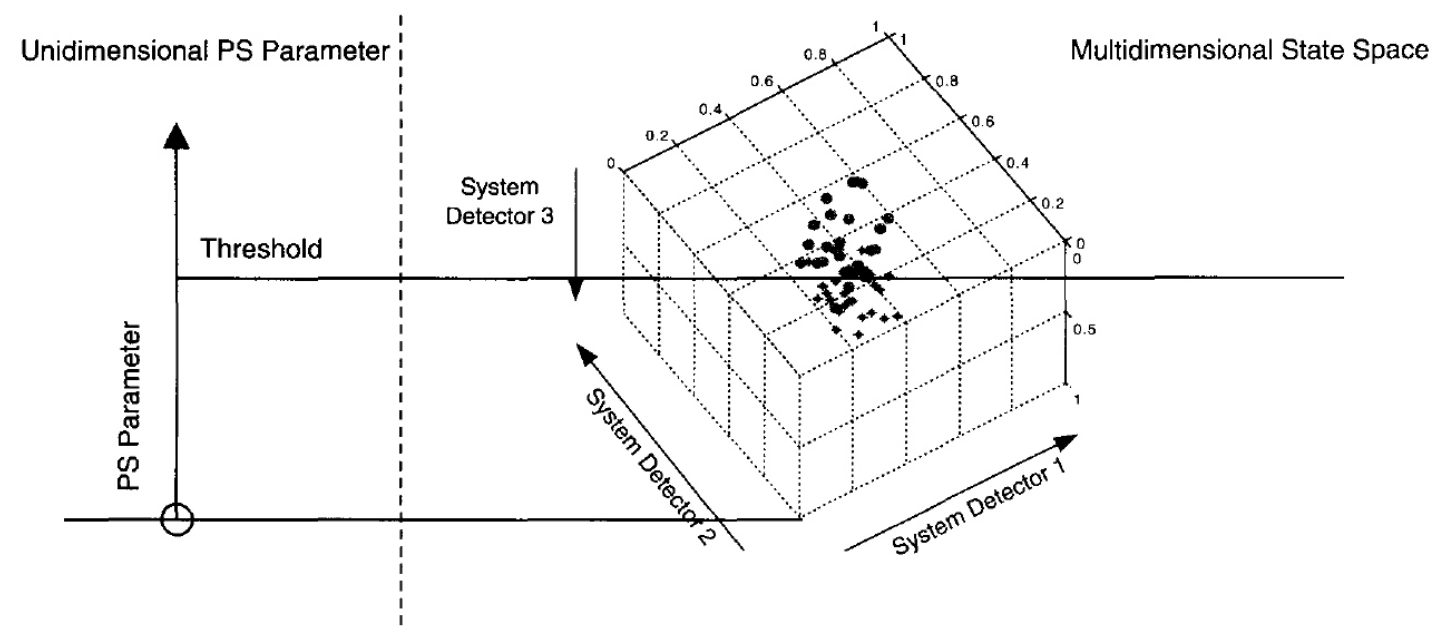

(b)

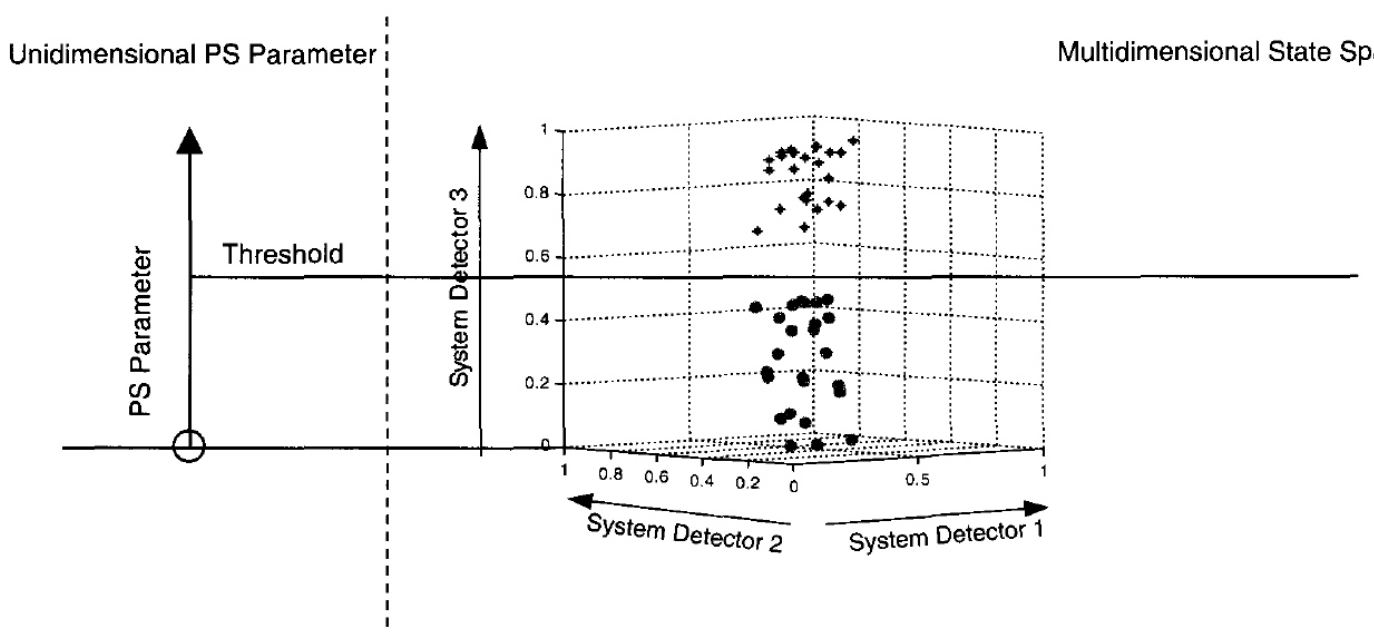

(c)

FIGURE 2 Effects of detector weights on TRPS state space: $(a)$ worst set of weights, $(b)$ better set of weights, and $(c)$ best set of weights. 
a better separation, and Figure 2c shows the best set of weights, which provides total separation of the two state distributions.

\section{Optimal Framework for TRPS System Design}

A comprehensive TRPS design approach for closed-loop systems was developed by Abbas et al. (15). The general framework can be summarized in the following steps:

1. Design the closed-loop system to address a wide range of traffic conditions (states). This design can be achieved by selecting several levels of traffic conditions and designing an appropriate timing plan for each level.

2. Group similar traffic states together (using clustering techniques) and selectarepresentativestatefromeachgroup.

3. Use a simulation program, such as $\operatorname{CaRS1M}(16)$, to simulate the closed-loop system with system detectors placed at all candidate approaches. Obtain the system measures of effectiveness (MOEs) and the system detectors' occupancy and counts for each simulated state.

4. Perform global system optimization to select the best $\mathrm{n}$ timing plans for overall system performance and assign each state to a timing plan (n could be up to the maximum number of timing plans that can be stored in the controllers).

5. Select system detectors that allow the best discrimination of different states. This objective can be achieved by using stepwise discriminant analysis (17).

6. Determine the weights associated with the selected system detectors such that the CC parameter calculated using these weights captures most of the variability between different states. This objective can be achieved by using canonical discriminant analysis (17).

7. Using the PS parameter calculated from the relevant CC parameters, obtain the discriminant functions that can distinguish between different states.

8. Plot the discriminant functions and determine the points of their intersections. These points of intersections define the TRPS thresholds for different states.

Selection of a limited set of timing plans for optimum system performance under a wide range of traffic conditions is discussed by Abbas et al. elsewhere (15). The focus here is on the robust configuration of TRPS factors, parameters, and thresholds for a given set of timing plans (namely, Steps 5 through 8 in the proposed framework). The application of the foregoing procedure to one closedloop system in Texas is described in the following sections.

\section{Odem Closed-Loop System}

A closed-loop system in adem, Texas, was studied and analyzed following the proposed methodology. The system consisted of three intersections operated with a video image vehicle detection system. The site was selected because the intersection video cameras could be used to record video and collect data over several days including one of the major holidays (Thanksgiving). The adem system is shown in Figure 3, which also shows the location

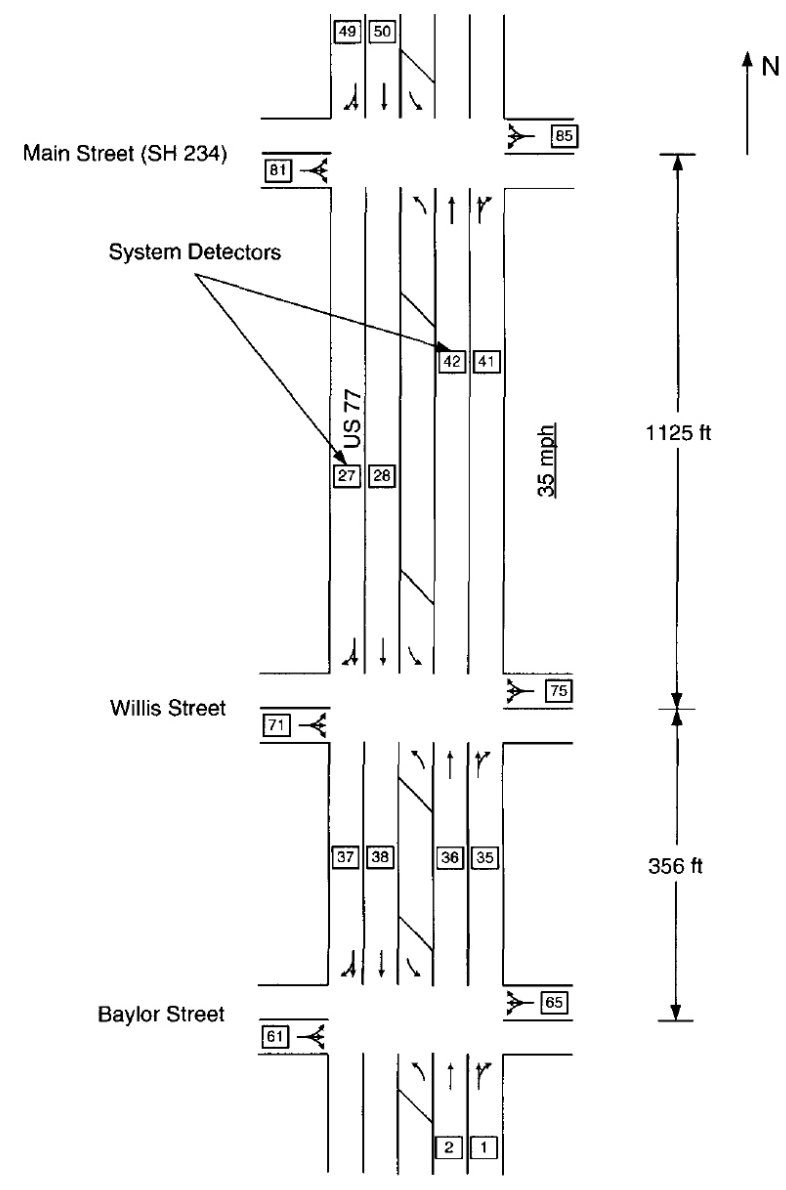

FIGURE 3 Odem closed-loop system network.

and identification of system detectors as they were later placed in the CORSIM network. These system detectors were placed $400 \mathrm{ft}$ upstream of each intersection, except for the system detectors between Willis and Baylor Streets, where the spacing between the intersections did not allow placing the detectors $400 \mathrm{ft}$ upstream. These specific detectors were placed midway between the two intersections.

\section{Timing Plans}

Table 1 shows the traffic states for the adem system. These states are representative of normal and holiday traffic conditions. Timing plans corresponding to each of these states were developed using Synchro 5.0 (18). The final plans are given in Table 2. Figure 4 shows the association of adem temporal traffic distribution with the designed timing plans (shown as $\mathrm{Pi}$ ). After the system detectors were placed in the adem CaRS1M network, simulations were performed for all plan-state combinations. Detector occupancy and count values were collected over 5-minute intervals for all of the simulation files.

\section{Discriminant Analysis}

Discriminant analysis is a Bayesian-based procedure in which previous knowledge of observation states is used 
TABLE 1 Traffic Volume States on Odem Network

\begin{tabular}{|c|c|c|c|c|c|c|c|}
\hline \multirow{2}{*}{ State } & \multirow{2}{*}{ Intersection } & \multicolumn{6}{|c|}{ Traffic Volume (vph) } \\
\hline & & EB & NB & NBL & WB & SB & SBL \\
\hline \multirow{3}{*}{1} & Baylor \& US77 & 56 & 847 & 11 & 85 & 1462 & 13 \\
\hline & Willis \& US77 & 50 & 1030 & 14 & 94 & 1742 & 1 \\
\hline & Main St \& US77 & 24 & 1305 & 22 & 52 & 1836 & 40 \\
\hline \multirow{3}{*}{2} & Baylor \& US77 & 61 & 828 & 9 & 15 & 1369 & 23 \\
\hline & Willis \& US77 & 60 & 926 & 15 & 64 & 1571 & 0 \\
\hline & Main St \& US77 & 33 & 1371 & 4 & 73 & 1969 & 24 \\
\hline \multirow{3}{*}{3} & Baylor \& US77 & 71 & 567 & 14 & 63 & 574 & 8 \\
\hline & Willis \& US77 & 46 & 620 & 17 & 52 & 688 & 4 \\
\hline & Main St \& US77 & 27 & 984 & 25 & 68 & 808 & 57 \\
\hline \multirow{3}{*}{4} & Baylor \& US77 & 21 & 199 & 0 & 14 & 100 & 0 \\
\hline & Willis \& US77 & 24 & 175 & 0 & 14 & 80 & 0 \\
\hline & Main St \& US77 & 18 & 261 & 1 & 19 & 131 & 9 \\
\hline \multirow{3}{*}{5} & Baylor \& US77 & 17 & 225 & 1 & 14 & 60 & 0 \\
\hline & Willis \& US77 & 21 & 187 & 0 & 14 & 80 & 0 \\
\hline & Main St \& US77 & 16 & 299 & 1 & 20 & 88 & 4 \\
\hline \multirow{3}{*}{6} & Baylor \& US77 & 70 & 494 & 26 & 62 & 429 & 9 \\
\hline & Willis \& US77 & 45 & 534 & 14 & 49 & 514 & 4 \\
\hline & Main St \& US77 & 33 & 883 & 38 & 66 & 607 & 61 \\
\hline
\end{tabular}

to formulate a discriminant function for each state. These discriminant functions, in tum, can be used to classify future observations into one of the known states. Predicting observation states with known classifications (e.g., resubstitution of original data) using the formulated discriminant functions can be used to estimate the rates of correct classifications, which are typically used to evaluate the performance of the discriminant functions.

Canonical discriminant analysis, however, is a dimensionality reduction technique similar to principal component analysis that can be used to determine the best linear combinations of variables such that the differences between classes are well defined.

Discriminant analysis and canonical discriminant analysis were used to obtain PS thresholds and TRPS detector weights, respectively.
Selection of System Detectors

One of the limitations imposed by the TRPS control mechanism implemented by traffic controller manufacturers is the maximum number of system detectors that can be assigned to each CC parameter. To design the TRPS system within this constraint, stepwise discriminant analysis was used to select a maximum of eight system detectors that have the most correlation with the changes in the state variable. Stepwise discriminant analysis is a procedure that iteratively includes or excludes a subset of quantitative variables to produce a good discrimination model. The final subset of system detectors is shown in Table 3. It should be noted that the final detectors selected were those at the entrances and exit of the closedloop system and one detector on one of the cross streets. This result was expected since these detectors capture significant changes in traffic states on the closed-loop system network.

TABLE 2 Designed Timing Plans on Odem Network

\begin{tabular}{|c|c|c|c|c|c|c|c|c|c|}
\hline \multirow{2}{*}{ Plan } & \multirow{2}{*}{ Intersection } & \multirow{2}{*}{ Cycle (sec.) } & \multicolumn{6}{|c|}{ Split (sec.) } & \multirow[b]{2}{*}{ Offset (sec.) } \\
\hline & & & SBL & NB & $\mathrm{EB}$ & NBL & SB & WB & \\
\hline \multirow{3}{*}{1} & Baylor \& US77 & 90 & 9 & 48 & 33 & 9 & 48 & 33 & 0 \\
\hline & Willis \& US77 & 90 & 9 & 48 & 33 & 9 & 48 & 33 & 83 \\
\hline & Main St \& US77 & 90 & 9 & 48 & 33 & 9 & 48 & 33 & 65 \\
\hline \multirow{3}{*}{2} & Baylor \& US77 & 70 & 10 & 34 & 26 & 9 & 35 & 26 & 0 \\
\hline & Willis \& US77 & 70 & 9 & 35 & 26 & 9 & 35 & 26 & 63 \\
\hline & Main St \& US77 & 70 & 9 & 36 & 25 & 9 & 36 & 25 & 40 \\
\hline \multirow{3}{*}{3} & Baylor \& US77 & 85 & 9 & 58 & 18 & 10 & 57 & 18 & 0 \\
\hline & Willis \& US77 & 85 & 9 & 58 & 18 & 9 & 58 & 18 & 82 \\
\hline & Main St \& US77 & 85 & 10 & 57 & 18 & 9 & 58 & 18 & 65 \\
\hline \multirow{3}{*}{4} & Baylor \& US77 & 125 & 9 & 86 & 30 & 9 & 86 & 30 & 0 \\
\hline & Willis \& US77 & 125 & 9 & 86 & 30 & 9 & 86 & 30 & 120 \\
\hline & Main St \& US77 & 125 & 9 & 86 & 30 & 9 & 86 & 30 & 8 \\
\hline \multirow{3}{*}{5} & Baylor \& US77 & 60 & 9 & 31 & 20 & 9 & 31 & 20 & 0 \\
\hline & Willis \& US77 & 60 & 9 & 31 & 20 & 9 & 31 & 20 & 55 \\
\hline & Main St \& US77 & 60 & 9 & 31 & 20 & 9 & 31 & 20 & 31 \\
\hline \multirow{3}{*}{6} & Baylor \& US77 & 115 & 9 & 62 & 44 & 9 & 62 & 44 & 0 \\
\hline & Willis \& US77 & 115 & 9 & 62 & 44 & 9 & 62 & 44 & 111 \\
\hline & Main St \& US77 & 115 & 9 & 62 & 44 & 9 & 62 & 44 & 105 \\
\hline
\end{tabular}




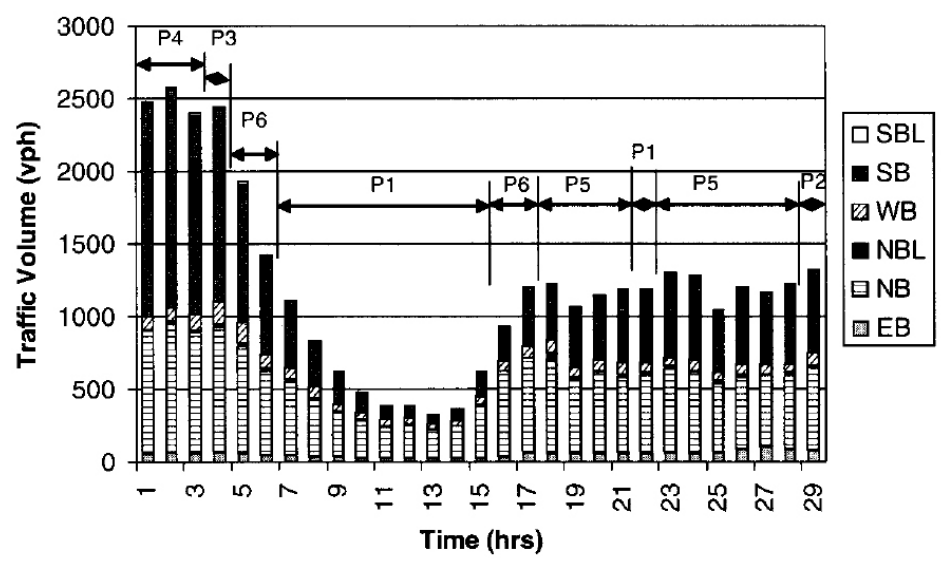

FIGURE 4 Assignment of timing plans to different traffic states.

\section{Determination of Detector Weights}

Detector weights were determined using canonical discriminant analysis. This procedure was especially important since each of the PS parameters (cycle, split, and offset) is calculated on the basis of a combination of system detectors. Theoretically, each PS parameter can have its own canonical variable such that the most differentiation power is achieved. In the Odem network, however, only the cycle-level parameter was needed. The SAS canonical discriminant procedure was used (19). Detector weights associated with each of the occupancies and counts are given in Table 3. It should be noted that final detector weights do not show negative values since negative values cannot be entered as weights in the current setup of traffic controllers (another limitation of the TRPS mechanism). The final discriminant functions were plotted using the modified weights to account for this limitation.

The count scaling factor is calculated in the controller as the raw volume divided by the maximum approach capacity as an input by the user. Since the analysis used volumes accumulated over $5 \mathrm{~min}$ as the sampling rate and the controller will convert the raw volume back to a volume per minute, the maximum approach capacity should be entered as $20(100 / 5)$ vehicles per minute. For example, if the raw volume over 5 min was 10 vehicles, the controller will divide that by the sampling period as $10 / 5=2$ vehicles per minute. The controller will then divide that by the maximum capacity of 20 vehicles per minute to arrive at $2 / 20=10 \%$, which is the value used in the analysis. The maximum occupancy rate should be kept at $100 \%$ since the controller will always interpret occupancy as a percentage rate. For example, if the raw occupancy over the 5-minute sampling period was 30\%, the controller will divide that by the maximum occupancy rate to arrive at $30 / 100=30 \%$.

\section{Determination of State Discriminant Functions}

Once the canonical variable coefficients were determined, discriminant analysis was performed on the new defined variable. That is, each observation in the data set had an associated state as well as a PS parameter value calculated as the summation of each system detector actuation multiplied by the final weight assigned to that detector. The results of the discriminant analysis are shown in Figure 5 , in which the misclassification rate for each state is shown. It should be noted that Figure 5 shows high crossclassification between States 1 and 2, which suggests that States 1 and 2 should be considered as one state to minimize the transitioning effect between the two. Similarly, States 3 and 4 should be considered as another state, and States 5 and 6 should be considered as the third state.

The similarity of States 1 and 2 as far as detector actuation is concerned is probably due to the actuated operation of the signal in CORSIM, which might not have been fully accounted for by Synchro when the timing plans were designed. This factor is evident in Table 4 , in which total control delay resulting from implementing Plan 2 with State I actually resulted in less delay than implementing Plan 1 with State 1 (which is

TABLE 3 System Detector Weights

\begin{tabular}{lclllc}
\hline System Detector & \multicolumn{2}{c}{ Raw Weight } & & \multicolumn{2}{c}{ Detector Weight } \\
\cline { 2 - 3 } \cline { 5 - 6 } Number & Count & Occupancy & & Count & Occupancy \\
\hline 2 & 0.136 & 0.095 & & 14 & 10 \\
38 & -0.006 & 0.006 & & 0 & 1 \\
61 & -0.063 & 0.007 & & 0 & 1 \\
42 & 0.039 & 0.007 & & 4 & 1 \\
50 & 0.072 & 0.149 & & 7 & 15 \\
\hline
\end{tabular}




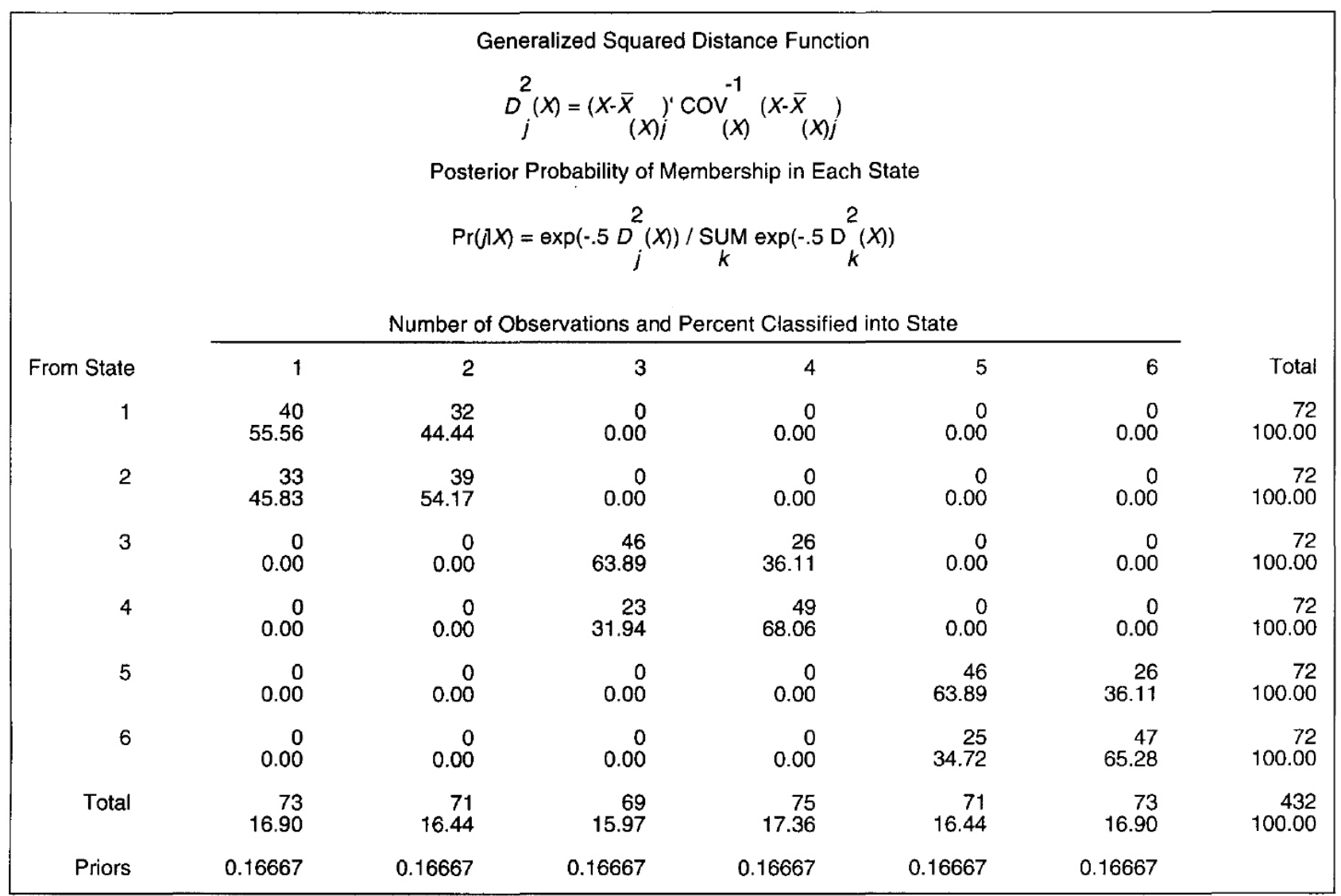

FIGURE 5 Output of SAS discriminant analysis.

supposed to be the optimal plan for State 1). Other entries in Table 4 support the same argument. It should be noted that the misclassification error from the suggested groups into other groups is $0 \%$. This finding means that if the six original states were treated as three states and were assigned timing plans accordingly, TRPS will achieve 100\% state identification accuracy.

\section{Threshold Selection}

Discriminant analysis results in determination of discriminant functions that can be used to determine the group to which every observation belongs. Figure 6 shows a plot of the discriminant functions for each state versus the cycle PS parameter, which clearly shows that the original six states are actually only three. For any PS parameter value, the discriminant function with the highest value determines the group to which the observation belongs. As such, the intersection points of the discriminant functions determine the PS parameter thresholds. The values shown in Figure 6 suggest that thresholds of 16 and 22 are needed to switch from Timing Plan 1 to Timing Plan 5 and from Timing Plan 5 to Timing Plan 3, respectively. The fact that using three states to represent the system results in $0 \%$ misclassification error means that there is no need to set up different entering and exiting thresholds.

TABLE 4 Total Delay in Vehicle Minutes for Combinations of States and Timing Plans

\begin{tabular}{l|llllll}
\hline \multirow{2}{*}{ State } & \multicolumn{6}{l}{ Timing Plan } \\
\cline { 2 - 7 } & 1 & 2 & 3 & 4 & 5 & 6 \\
\hline 1 & 159923 & 144345 & 860351 & 797163 & 146272 & 187125 \\
2 & 174956 & 147681 & 890592 & 807475 & 169938 & 202559 \\
3 & 142325 & 146893 & 146963 & 138874 & 194654 & 145279 \\
4 & 162874 & 156435 & 141550 & 152474 & 195355 & 157304 \\
5 & 164547 & 136740 & 545243 & 187995 & 134028 & 182488 \\
6 & 191926 & 160047 & 882086 & 803113 & 155608 & 225721 \\
\hline
\end{tabular}

NotE: Shaded cell in each row identifies the timing plan that results in minimum control delay for the particular traffic state. 


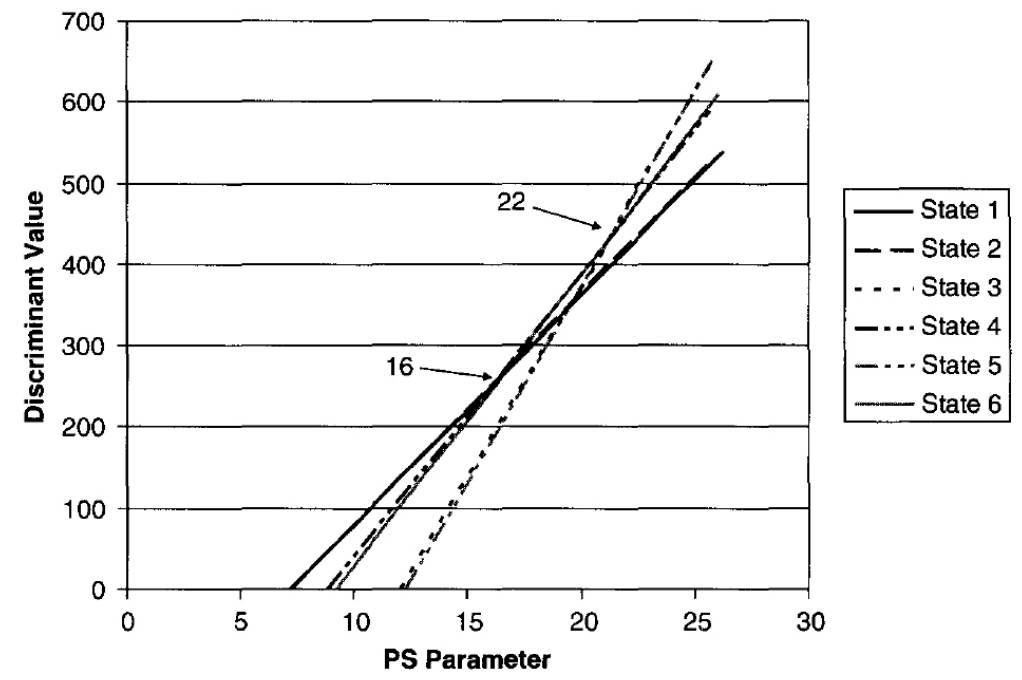

FIGURE 6 TRPS threshold determination.

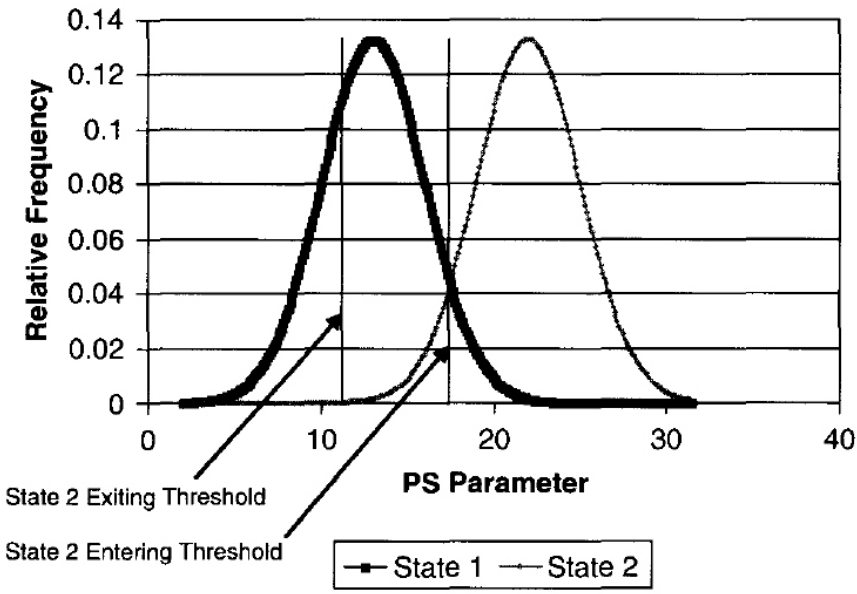

(a)

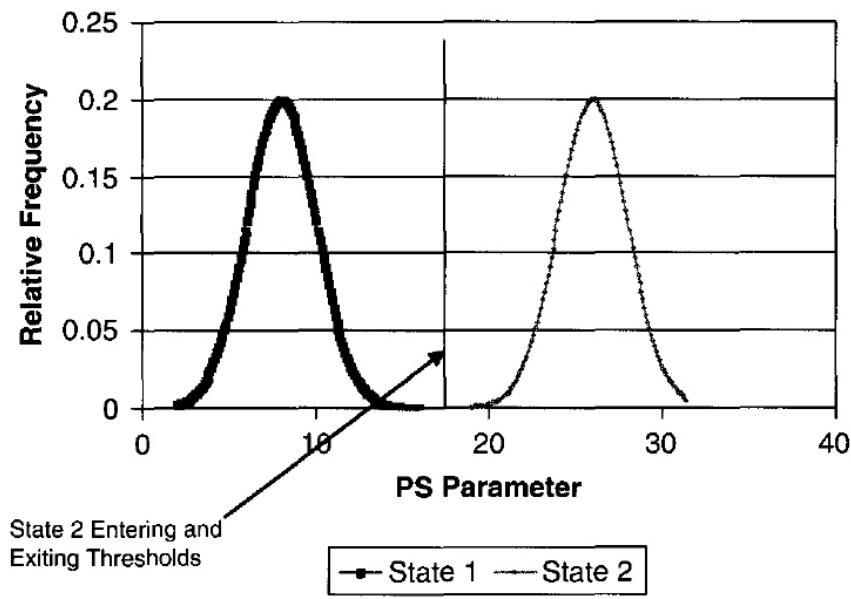

(b)

FIGURE 7 Conceptual illustrations of entering and exiting thresholds: (a) states with cross-classification errors; (b) states without cross-classification errors.
As shown in Figure 7, different entering and exiting thresholds are only needed when state distributions are not mutually exclusive. Figure 7a shows a conceptual case of two states with cross-classification errors. It should be noted that in such a case, an exiting threshold needs to be set lower than the entering threshold to achieve a stable operation. Figure $7 \mathrm{~b}$ shows mutually exclusive state distributions in which the exiting threshold could be set equal to the entering threshold without the system stability's being affected.

\section{CONCLUSION}

Although the TRPS mode has the greatest potential to provide an optimal operation utilizing existing capabilities of closed-loop systems, it remains an untapped resource for lack of system setup guidelines. A new robust methodology for the selection of TRPS optimal parameters and thresholds using Bayesian-based discriminant analysis is outlined. The methodology proposes that timing plans only be assigned to distinct states. The proposed methodology was tested using field data from a closed-loop system in Odem, Texas, and achieved 100\% classification accuracy.

\section{ACKNOWLEDGMENTS}

This work was sponsored by the Texas Department of Transportation (DOT) and was conducted for Texas DOT's Research and Technology Implementation Office. The materials and methods presented were developed as part of Texas DOT Project 0-4421, “A Simplified Approach for Selecting Optimal Traffic Responsive Control Parameters." The authors recognize the project director, Brian Van De Walle, for his support and guidance throughout the duration of this project.

\section{REFERENCES}

1. Meyer, M. D. A Toolbox for Alleviating Traffic Congestion and Enhancing Mobility. Institute of Transportation Engineers, Washington, D.C., 1997. 
2. Fambro, D. B., S. M. Sangineni, C. A. Lopez, S. R. Sunkari and R. T. Barnes. Benefits ofthe Texas Traffic Light Synchronization (TLS) Grant Program II. Research Report 301O-IF, Vol. 2. Texas Transportation Institute, College Station, 1995.

3. Henry, R. D., R. A. Ferlis and J. L. Kay. Evaluation of UTCS Control Strategies-Technical Report. Report FHWARD-76-150. FHWA, U.S. Department of Transportation, Aug. 1976.

4. Kay, J. L., J. C. Allen and J. M. Bruggeman. Evaluation of the First Generation UTCS/BPS Control Strategy. Vol. I, Technical Report. Report FHWA-RD-75-27. FHWA, U.S. Department of Transportation, Feb. 1975.

5. Henry, R. D., R. A. Ferlis and R. M. White. Application of UTCS First Generation Control Software in New Orleans. Report FHWA-RD-78-3. FHWA, U.S. Department of Transportation, Jan. 1978.

6. Special Report 209: Highway Capacity Manual, 3rd ed. (1994 update). TRB, National Research Council, Washington, D.C., 1994.

7. Hanbali, R. and C. J. Fornal. Methodology for Evaluating Effectiveness of Traffic-Responsive Systems on Intersection Congestion and Traffic Safety. Transportation Research Record 1603, TRB, National Research Council, Washington, D.C., 1997, pp. 137-149.

8. Nelson, E., M. Abbas, G. Shoup and D. Bullock. Development of Closed Loop System Evaluation Procedures. Report FHWA1IN/JTRP. FHWA, U.S. Department of Transportation, May 2000.

9. Kay, J., R. Henry and S. Smith. Locating Detectorsfor Advanced Traffic Control Strategies: Handbook. Report FHWARD-75-91. FHWA, U.S. Department of Transportation, 1975.
10. Woods, D. and N. Rowan. Detector Locationfor Computerized Arterial Street Sampling Detectors. Report FHWA/TX9511392-6. Texas Transportation Institute, Texas A\&M University System, College Station, 1995.

11. Master Controller Software. Naztec, Inc., Sugarland, Tex., Aug. 1992.

12. ASC/2M-1000 Zone Master Programming Manual. Econolite Control Products, Inc., Anaheim, Calif., n.d.

13. MARC 300 Series Master Controller. Automatic Signal/Eagle Signal, Austin, Tex., n.d.

14. Balke, K. N., R. S. Keithireddipalli and C. L. Brehmer. Results of Simulation Studies Relating to the Operation of ClosedLoop Systems in a Traffic Responsive Mode. Research Report 2929-2, Project 7-2929. Texas Transportation Institute, College Station, Jan. 1997.

15. Abbas, M. M., N. A. Chaudhary, A. Sharma, S. P. Venglar and R. J. Engelbrecht. Methodology for Determination of Optimal Traffic Responsive Plan Selection Control Parameters. Research Report 4421-1. Texas Transportation Institute, College Station, Sept. 2003.

16. CORS 1M User's Manual. ITT Systems \& Sciences Corporation; FHWA, U.S. Department of Transportation, 2003.

17. Huberty, C. J. Applied Discriminant Analysis. John Wiley \& Sons, Inc. New York, 1994.

18. Trafficware. Synchro 5.0, Traffic Signal Timing Software. Albany, Calif., 2000.

19. SAS/STAT User's Guide, Version 8.1. SAS Institute Inc., Cary, N.C., 2001.

Publication of this paper sponsored by Traffic Signal Systems Committee. 